\title{
Erratum to: Neurogenic muscle cramps
}

\author{
Hans D. Katzberg ${ }^{1,2,3}$
}

Published online: 6 January 2016

(C) Springer-Verlag Berlin Heidelberg 2015

\section{Erratum to: J Neurol (2015) 262:1814-1821 DOI 10.1007/s00415-015-7659-x}

The author would like to correct the errors in the publication of the original article. The corrected details are given below for your reading.

In the results section, the sixth sentence in the second paragraph of the Pharmacotherapy (Table 3) section should read as:

A single study found tetrahydrocannabinol (THC) was not effective in preventing muscle cramps in patients with ALS.

As such, the THC agent should not be included in Table 3. The correct Table 3 is given in the following page.

The online version of the original article can be found under doi:10.1007/s00415-015-7659-x.

Hans D. Katzberg

hans.katzberg@utoronto.ca

1 University of Toronto, Toronto, Canada

2 University Health Network, Toronto, Canada

3 Toronto General Hospital/UHN, 200 Elizabeth Street, 5ES-306, Toronto, ON M5G 2C4, Canada 
Table 3 Pharmacological treatments for muscle cramps according to level of evidence

\begin{tabular}{|c|c|c|c|}
\hline Medication & Dose & $\begin{array}{l}\text { Class of } \\
\text { evidence }\end{array}$ & Adverse events $^{\mathrm{e}}$ \\
\hline $\begin{array}{l}\text { Quinine sulfate } \\
\text { [54-56] }\end{array}$ & $150-450 \mathrm{mg}$ daily $(300 \mathrm{mg})^{\mathrm{a}}$ & I & $\begin{array}{l}\text { Cinchonism, bitter taste, arrhythmia, ITP, TTP, rash, thrombocytopenia, } \\
\text { nausea, blurry vision }\end{array}$ \\
\hline $\begin{array}{l}\text { Vitamin } \\
\text { B-complex [48] }\end{array}$ & $\begin{array}{l}\text { Includes } 50 \mathrm{mg} \text { of B1, B2, B3, } \\
\text { B6, B12 daily }\end{array}$ & II & Caution with B6 hyper-vitaminosis which can cause neuropathy \\
\hline Diltiazem [60] & $30 \mathrm{mg}$ daily & II & Edema, headache, nausea, dizziness, rash, asthenia, arrhythmia \\
\hline $\begin{array}{l}\text { Natridrofuryl } \\
\text { oxalate }^{c}[67]\end{array}$ & $300 \mathrm{mg}$ twice per day & II & $\begin{array}{l}\text { Diarrhea, nausea, vomiting skin rash, elevation in liver enzymes, renal } \\
\text { calculi }\end{array}$ \\
\hline Mexiletine [66] & Up to $300 \mathrm{mg}$ daily & IV & $\begin{array}{l}\text { Nausea, vomiting, heartburn, ataxia, dizziness, tremor, palpitation, } \\
\text { hypotension, blurry vision, arrythmia }\end{array}$ \\
\hline $\begin{array}{l}\text { Carbemazepine }^{\mathrm{d}} \\
\text { [43] }\end{array}$ & Maximum $1,600 \mathrm{mg}$ per day & IV & $\begin{array}{l}\text { Blurry vision, confusion, dizziness, nausea, drowsiness, Steven-Johnson } \\
\text { Syndrome, SIADH, hyponatremia }\end{array}$ \\
\hline $\begin{array}{l}\text { Leveteracitam } \\
\text { [63] }\end{array}$ & $1,500 \mathrm{mg}$ twice per day & IV & Fatigue, headache, insomnia, arthralgia, edema \\
\hline
\end{tabular}

TTP thrombotic thrombocytopenia purpura, ITP idiopathic thrombocytopenic purpura, SIADH syndrome of inappropriate antidiuretic hormone secretion

${ }^{\text {a }}$ Federal Drug Administration warning against use for treatment of muscle cramps in the United States

b Studied in amyotrophic lateral sclerosis

c Not routinely available in the United States

d Studied in cramp-fasciculation syndrome

e Common and serious adverse events reported with medications, not limited to muscle cramp trials 